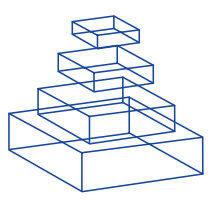

\title{
Bridging multiple levels of exploration: towards a neuroengineering-based approach to physiological and pathological problems in neuroscience
}

\author{
Laura Ballerini \\ University of Trieste, Italy \\ Correspondence: ballerin@psico.units.it
}

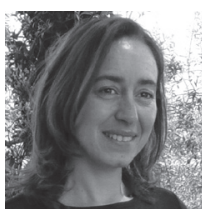

Laura Ballerini graduated (M.D.) at the Università di Firenze, Italy in 1988. She was a post-doc at UCL from 1991 and later became assistant professor in Physiology at the Biophysics Sector of the International School for Advanced Studies of Trieste, Italy in 1995. Since 2002 she is associate professor of Physiology at the Università di Trieste, Italy. Laura Ballerini has provided important contribution to the understanding of spinal network physiology, plasticity and development. Since 2004 she is involved in a research program to exploit nanotechnology research in the central nervous system and since 2006 she coordinates an $E U$ project designed to use the convergence of nanotechnology and neurobiology to develop novel biotechnologies that may open a new frontier for brain research and directly or indirectly aid in CNS repair.
Neuroengineering is a rapidly growing discipline that takes its lymph from the increasing cross-fertilization of many areas of technology and science. For example, by means of neuroengineering, advances in diverse technologies and in cellular and molecular biology converge into powerful tools to improve our understanding and treatment of neural (dis)functions. Recently such a discipline has gone beyond the concept of a simple application of engineering principles to central nervous system (CNS) comprehension, leading to the emergence of one of the more exciting interdisciplinary research fields in modern neuroscience. Neuroengineering applies novel approaches to the study of neuroscience by bringing together tools from computational neuroscience, information theory, electronics, electrophysiology, biomaterials, nanotechnologies and tissue engineering, towards understanding, repairing, replacing, enhancing, and exploiting the electrical properties of the nervous system. This cross-modality might be at times a source of confusion. To better clarify this research strategy let us take the example of computational neuroscience. In neuroengineering the use of computational neuroscience is explicit and aimed at consolidating quantitative tools for the reverse engineering of molecular, synaptic and cellular bases of neural systems. Computational tools are a key component of neuroengineering, as mathematics and construction theory are fundamental instruments for engineers to build machines.

Understanding the brain is one of the grand challenges in neuroscience research. In neuroengineering such challenge extends from fundamental research on CNS computation to new frontiers in neural prosthetics. Neuroengineering benefits therefore of a unique intellectual position that allows the use of experimental and theoretical tools to study issues ranging from memory formation or sensory encoding, brain injury and repair to diagnostic technology and prosthesis, or theoretical neuroscience. One of the striking features of neuroengineering is that, this discipline although crucial in clinical strategies aimed at gaining functional recovery, easily shifts its sphere of opportunities to general issues, including understanding crucial questions in neuroscience, such as the translation of molecular events in the CNS into complex behaviors. More specifically, we can indicate the following as primary areas of interest of neuroengineering: 


\section{REFERENCES}

Zhang, F., Aravanis, A. M., Adamantidis, A., de Lecea, L., Deisseroth, K. (2007). Circuitbreakers: optical technologies for probing neural signals and systems. Nat Rev Neurosci. 8, 577-81.

Silver, R., Boahen, K., Grillner, S., Kopell, N., Olsen, K. L. (2007). Neurotech for neuroscience: unifying concepts, organizing principles, and emerging tools. J. Neurosci. 27, 11807-11819.

Gradinaru, V., Thompson, K. R., Zhang, F., Mogri, M., Kay, K., Schneider, M. B., Deisseroth, K. (2007). Targeting and readout strategies for fast optical neural control in vitro and in vivo. J. Neurosci. 27, 14231-14238.

Stieglitz, T. (2007). Restoration of neurological functions by neuroprosthetic technologies: future prospects and trends towards micro-, nano-, and biohybrid systems. Acta Neurochir. Suppl. 97, 435-42.

i) to develop new (nano)technological tools to probe the brain at various scales (i.e. from genes to synapses; from cells to networks; from systems to artificial systems), ii) to design and implement novel devices to replace parts of the brain; iii) to consolidate a design-oriented approach to the understanding and development of new methods to provide brain stimulators (DBS, optical stimulation) as well as chemical repair and delivery/release systems.

A general example of the leading role of neuroengineering in novel approaches to neuroscience is that of the microelectromechanical systems (MEMS), which exploits the technologies used by the integrated-circuit industry to design and fabricate new classes of micro- and nano-scale devices, such as microsensors, microactuators, and Microsystems. The increasing leadership of Neuroengineers in this technology is paving the road for the development of devices for interfacing with the nervous system at unprecedented small scales and complexity. Within this framework, neuroengineering rapidly converged with novel disciplines such as nanotechnology. Nanotechnology is an area of science and engineering that uses physical or chemical methods to manipulate and control materials and devices at the molecular level. Neuroengineering challenges nanotechnology applications can significantly improve the understanding of how the nervous system works, how it fails in disease and how we can intervene at a molecular level. Such challenges include several ambitious goals to be pursued in the next decades, which can be categorized into three major objectives: 1) the design of new generation-interfaces between an artificial system and the nerve tissue, able to perform bidirectional communication at different levels: molecular, synaptic and cellular ones; 2) the achievement of computational descriptions of the performance of hybrid artificial-neural systems; 3 ) the design of smart materials triggering specific molecular, synaptic and cellular reorganization in a CNS network, aiming at an endogenous and material-driven accurate manipulation of the neural system. Ultimately, the potential of neuroengineering endeavor includes the challenge of generating a novel unified scientific language that allows traditional and non-traditional scientific disciplines to work together.

Silva, G. A. (2007). Nanotechnology approaches for drug and small molecule delivery across the blood brain barrier. Surg Neurol. 67, 113-6.

Pearce, T. M., Williams, J. C. (2007). Microtechnology: meet neurobiology. Lab Chip. 7, 30-40.

Lebedev, M. A., Nicolelis, M. A. (2006). Brainmachine interfaces: past, present and future. Trends Neurosci. 29, 536-46.

Silva, G. A. (2006). Neuroscience nanotechnology: progress, opportunities and challenges. Nat. Rev. Neurosci. 7, 65-74.

Bellamkonda, R. V. (2006). Peripheral nerve regeneration: an opinion on channels, scaffolds and anisotropy. Biomaterials. 27, 3515-8.

Chapin, J. K. (2004). Using multi-neuron population recordings for neural prosthetics. Nat Neurosci. 7, 452-5.
Hu, X. L., Zhang, Y. T., Yao, J. (2002). Neuroengineering modeling of single neuron and neural interface. Crit Rev Biomed Eng. 30, 219-48.

Buettner, H. M. (1995). Neuroengineering in biological and biosynthetic systems. Curr Opin Biotechnol. 6, 225-9.

Front. Neurosci. (2008) 2, 1: 24-25. doi: 10.3389/ neuro.01.024.2008

Copyright (c) 2008 Ballerini. This is an openaccess publication subject to an exclusive license agreement between the authors and the Frontiers Research Foundation, which permits unrestricted use, distribution, and reproduction in any medium, provided the original authors and source are credited. 